\title{
A New Approach for Single Image Haze Removal
}

\author{
Jian-Qiang Lu, Wei-Xing Wang \\ College of Electronic Engineering, South China \\ Agricultural University, China \\ Guangdong Engineering Research Center for Monitoring \\ Agricultural Information, China \\ Key Laboratory of Information Acquisition and \\ Application in Agriculture, Guangzhou Science \\ Technology and Innovation Commission, China \\ E-mail: Ljq@scau.edu.cn,weixing@scau.edu.cn
}

\author{
De-Wei Huang, Ke-Xin Chen \\ College of Electronic Engineering, South China \\ Agricultural University, China \\ E-mail: huangdewei@stu.scau.edu.cn, \\ 285078375@qq.com
}

\begin{abstract}
We require the system for haze removal can process in real-time and have an accurate result. However, the existing algorithm can hardly meet the two requirements which we discuss above. In this paper, we propose a novel algorithm which is using the minimum channel in $R, G, B$ and the difference of the three channel that we refer before to estimate the transmission map. We assume that there is some relationship between transmission map and the minimum channel and the difference of the three channel. After having done a large number set of statistics, we find that they find the relation between them and make up a model to estimate the transmission map. After we get the transmission map, we can use the atmospheric scattering model to restore the image. After having done a lot of test, we find that our method do efficiency in haze removal.
\end{abstract}

Keywords-estimate transmission map; haze removal; atmospheric scattering model

\section{INTRODUCTION}

Outdoor images taken in bad weather usually had lost contrast and become blur which is resulting from the fact that light is absorbed and scattered by the turbid medium such as particles and water droplets in the atmosphere during the process of propagation [1]. The technology of computer vision is applying in a lot of field and it strongly depend on the definition of the input image. However, it would fail to work if the image is blur. Therefore, image dehazing as a pre-processing restoration step will benefit various algorithms that require image analysis.

Image dehazing can be divided into two kinds. One is that dehazing based on the enhancement of histogram [2-4]. It can use traditional algorithms to process hazy image such as enhance contrast and histogram equalization so that we can gain useful message from the processed image. It will lose a lot of message because this kind of algorithms don't concern about textural features of the original image. The other kind is dehazing based on atmospheric scattering model [5] which remove haze clearly and retain almost all message of the original image.

Both multiple images dehazing[6,7,8] and single image dehazing are based on atmospheric scattering model. Multiple images dehazing need images which is taken in different conditions so that it cannot process in real-time. Recently, most of us are concern about single image dehazing. Tan [9] maximum the local contrast of the image based on Markov Random Field (MRF) to gain clear image, but the image is always over-saturated. Fattal[10] is based on Independent Component Analysis to remove haze, but they failed while the haze is dense. With a large number set of statics, He et al.[11,12] propose dark channel prior (DCP) that in the non-sky area, at least one color channel has some pixels whose intensities are very low and trend to zero. They make a big progress in haze removal because this approach is simple and effective. However, they may fail when image has large area of sky or white scene. Guided filtering is proposed $[13,14]$ to improve the dark channel later. Tarel et al.[15] make an approach which is based on the median filter, but this approach cannot handle the edge of the image.

In this paper, we propose a novel algorithm which is using the minimum channel in $R, G, B$ and the difference of the three channel to estimate the transmission map. After having done a large number set of statistics, we find that our approach can remove the haze for the image clearly and in real-time.

\section{ATMOSPHERIC SCATTERING MODEL}

In order to describe hazy image, McCartney proposed atmospheric scattering model in 1976 which is shown in figure 1 and it can simplify as equation (1) and (2):

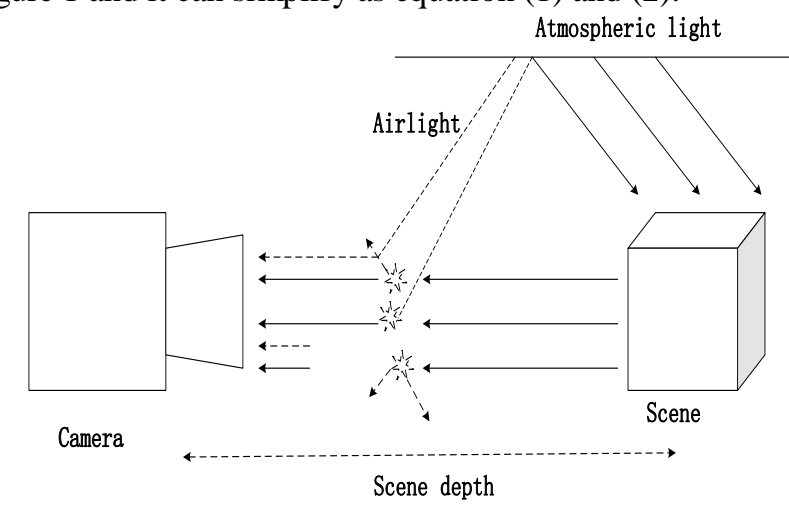

Figure 1. Atmospheric scattering mode.

$$
I(x)=J(x) t(x)+A(1-t(x))
$$




$$
t(x)=e^{-\beta d(x)}
$$

where $\mathrm{I}$ is the hazy image, $\mathrm{J}$ is the clear image that we wanted, $\mathrm{A}$ is the atmospheric light, $\mathrm{t}$ is the transmission, $\beta$ is the scattering coefficient of the atmosphere and $d$ is the depth of scene. We can change equation (1) into equation (3):

$$
J(x)=\frac{I(x)-A}{t(x)}+A
$$

Since I is known and our goal is the restore J so we must estimate $\mathrm{A}$ and $\mathrm{t}$ from the input image $\mathrm{I}$.

we define the minimum channel image $W(x)$ and difference between $R, G, B$ channel $D(X)$ which is shown in equation (4) and (5):

$$
\begin{gathered}
W(x)=\min _{C \in\{R, G, B\}} I^{C}(x) \\
D(x)=\frac{\sum_{C \in\{R, G, B\}} I^{C}-3 W(x)}{3}
\end{gathered}
$$

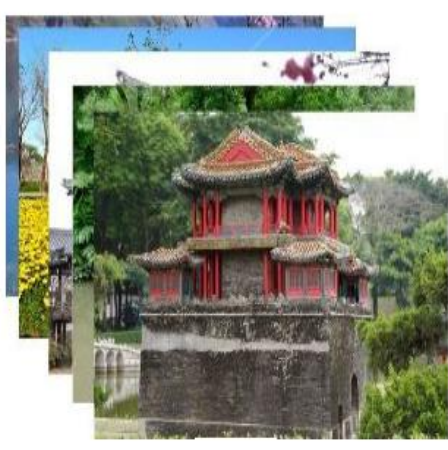

a
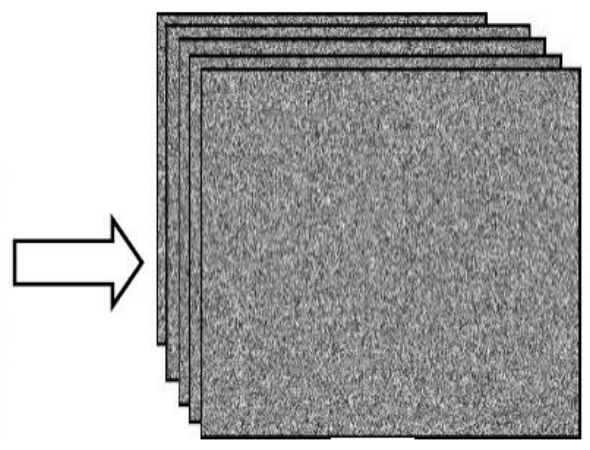

b
According to [15], we know that $\mathrm{W}(\mathrm{x})$ is have a lot of textural features so we try to use $W(x)$ and $D(x)$ to estimate transmission.

\section{ESTIMATE TRANSMISSION}

\section{A. Synthesis Hazy Image}

We can make an assumption that transmission is positively correlated with $\mathrm{W}(\mathrm{x})$ and $\mathrm{D}(\mathrm{x})$ and we have:

$$
t(x) \propto D(x)-W(x)
$$

It is very hard to estimate the coefficients because we can hardly get an accurate transmission from a single hazy image. Therefore, we prepare the training sample by synthesis transmission and atmospheric light randomly and calculate the hazy image by equation (1). we range the transmission in $(0,1)$ and the atmospheric light in $(0.85,1)$. The process of generating the training samples with haze-free images is shown in figure 2 :

Figure 2. The process of generating the training samples with haze-free images a: haze-free image;b: generated random transmission;c: the generated hazy images.

\section{B. Learning the Coefficients}

Based on the assumption, we can create a linear model as follows:

$$
t(x)=\theta_{0} W(x)+\theta_{1} D(x)+\theta_{2}
$$

In order to learn the coefficients, we minimum the following squared loss function[2]:

$$
L=\frac{1}{n|\omega|} \sum_{j=1}^{\omega_{i}}\left(t_{r i}(x)-\left(\theta_{0} W_{i}\left(x_{j}\right)+\theta_{1} D_{i}\left(x_{j}\right)+\theta_{2}\right)\right)
$$

where $n$ is the number of the training samples, $|\omega|$ is the total number of the pixels of all the haze images. we can learn the coefficients by equation (9):

$$
\frac{\partial \boldsymbol{L}}{\partial \boldsymbol{O}_{i}}=\mathbf{O}
$$

We use 500 training sample to learn the coefficient and we chose the best result as our training coefficients which is that $\theta_{0}=-0.8328, \theta_{1}=0.7485, \theta_{2}=0.8894$. Once we have the coefficients, we can calculate the transmission easily. These parameters will be used for recovering the transmission of the hazy image in this paper.

\section{ESTIMATION OF ATMOSPHERIC LIGHT}

We always choose the brightest pixel as the atmospheric light because sky area is always brighter than others. 
However, it would fail when there has no sky area or has white scene. In He's[12] approach, they pick the top 0.1 percent brightest pixels in the dark channel and select the highest intensity in the input image among these 0.1 percent pixels. This approach would also fail when there has large area of white scene. Therefore, we preprocess the input image before we estimate the atmospheric light. We mark the top 0.5 highest intensity pixels in the input image and we exclude these pixels when we use He's approach to estimate the atmospheric light.

\section{V.RESTORE THE HAZY IMAGE}

We can gain the transmission and the atmospheric light according what we have done above and we can restore haze-free image by equation (3). we notice that transmission occur as denominator and that it may have very low value which closed to zero. As the result, the restored image would have noise so we should give a lowest threshold for transmission. We follow by He's [12] proposal that set 0.1 as the lowest threshold and the change equation (3) to equation (10):

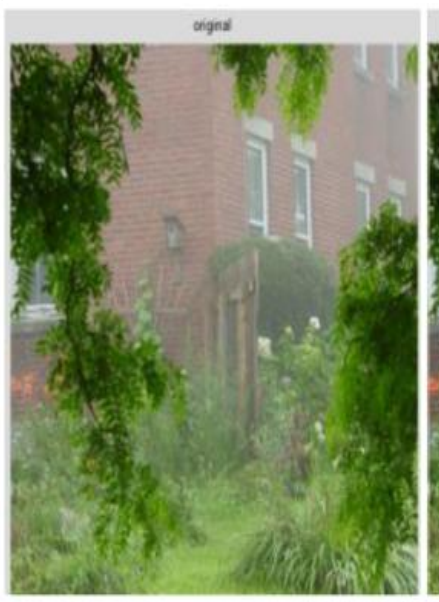

$$
J(x)=\frac{I(x)-A}{\max (0.1, t(x))}+A
$$

We use equation (10) to restore the haze-free image in this paper;

\section{Experimental Results}

We use computer with Intel(R) Core(TM) i3-2120 CPU @3.30GHz , 8G RAM do our experiment, and the processing software is MATLAB R2014a.

\section{A. Processing Result}

As the result are shown in Fig. 3. and Fig. 4., we can know that our approach does well in haze removal. Compare with dark channel, our approach gains more clear image and retain more information while compare with Tarel's approach.

Figure 3. A: Input image; b: dark channel; c: Tarel's approach; d: ours.

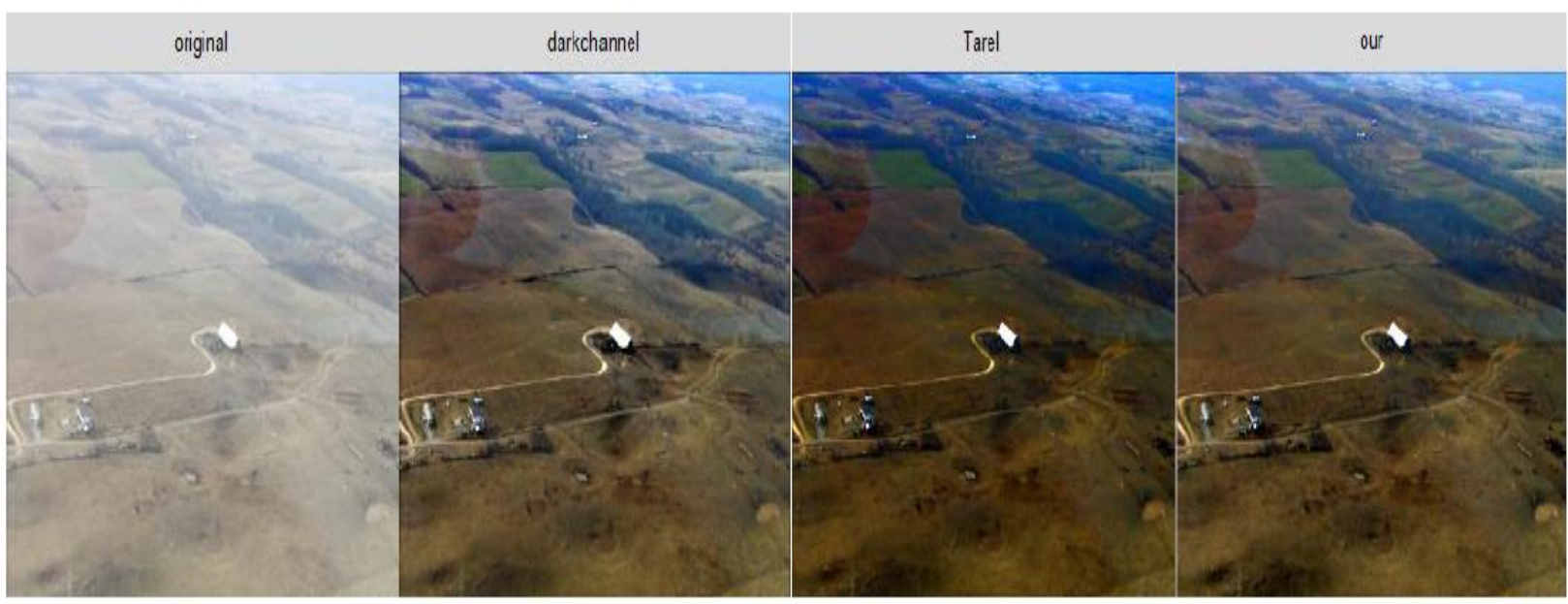

Figure 4. Input image; b: dark channel; c: Tarel's approach; d: ours. 


\section{B. Running Time}

We find that our approach is simple and efficiency. As shown in table 1, our approach process faster that it more suitable for the real-time system.

TABLE I. RUNNING TIME OF EACH APPROACH

\begin{tabular}{ccc}
\hline & Fig. 3. & Fig. 4. \\
\hline Size of input image & $450 * 441$ & $250 * 300 \mathrm{~s}$ \\
He's approach & $0.616229 \mathrm{~s}$ & $0.301966 \mathrm{~s}$ \\
Tarel' approach & $0.334899 \mathrm{~s}$ & $0.196347 \mathrm{~s}$ \\
Our approach & $0.297799 \mathrm{~s}$ & $0.174327 \mathrm{~s}$ \\
\hline
\end{tabular}

\section{CONCLUSION}

In this paper, we propose a novel approach for dehazing which is using the minimum channel and difference between its three color channel to estimate transmission. Our approach is simple and efficiency and it is more suitable for the real-time system. we can gain a clear image and have faster processing speed with our approach. There has disadvantage in our approach that the transmission is not very accurate by our estimation. Therefore, we hope that we can find a more accurate relationship between $\mathrm{t}(\mathrm{x}), \mathrm{W}(\mathrm{x})$ and $\mathrm{D}(\mathrm{x})$ in the future.

\section{ACKNOWLEDGEMENT}

This research was financially supported by National Nonprofit Industry Research (Funded by the Ministry of land and resources: 201411019).

\section{REFERENCES}

[1] S.G. Narasimhan, S.K. Nayar. Vision and the Atmosphere [J].
International Journal of Coumputer Vision, 2002, 48(3):233-254.

[2] Di W U, Zhu Q S. The Latest Research Progress of Image Dehazing $[J]$. Acta Automatica Sinica, 2015. (in Chinese)

[3] Stark J A, Fitzgerald W J. An alternative algorithm for adaptive histogram equalization[J]. Graphical Models and Image Processing, 1996, 58(2): 180-185.

[4] Stark J A. Adaptive image contrast enhancement using generalizations of histogram equalization[J]. Image Processing, IEEE Transactions on, 2000, 9(5): 889-896.

[5] E. J. McCartney. Optics of the atmosphere: scattering by molecules and particles. New York,John Wiley and Sons, Inc. 1976.

[6] Narasimhan S. G., Nayar S. K., Chromatic Framework for Vision in Bad Weather, IEEE conference on Computer Vision and Pattern Recognition, 2000, 1, 598-605

[7] Schechner Y. Y., Narasimhan S. G., Nayar S. K., Instant de-hazing of images using polarization, IEEE conference on Computer Vision and Pattern Recognition, 2001, 1, 325-332.

[8] S. Shwartz, E. Namer, Y. Y. Schechner. Blind haze separation, In Proc. CVPR, volume 2, pages 1984-1991, 2006.

[9] R. T. Tan. Visibility in bad weather from a single image. In Proc. CVPR, 2008

[10] R. Fattal. Single image dehazing. ACM Transactions on Graphics, 27(3), 2008

[11] K. He, J. Sun, and X. Tang. Single image haze removal using dark channel prior. In Preoccupy, 2009.

[12] K. He, J. Sun, and X. Tang. Single image haze removal using dark channel prior. IEEE

TPAMI 33(12): 2341-2353, 2011.

[13] K. He, J. Sun, and X. Tang. Guided image filtering. In Proc. ECCV, pages 1-14, 2010.

[14] K. He, J. Sun and X. Tang. Guided image filtering. IEEE TPAMI, 35(6): 1397-1409, 2013.

[15] J. P. Tarel, and H. Nicolas. Fast visibility restoration from a single color or gray level image. In Proc. ICCV, 2009. 